\title{
In Situ Nano-thermo-mechanical Experiment Reveals Brittle to Ductile Transition in Si Nanowires
}

\author{
Guangming Cheng ${ }^{1}$ and Yong $\mathrm{Zhu}^{2}$ \\ ${ }^{1}$ Princeton University, Princeton, New Jersey, United States, ${ }^{2}$ North Carolina State University, Raleigh, \\ North Carolina, United States
}

Silicon nanostructures have been used extensively in modern microelectronics. The ever-increasing integration density in microelectronic chips inevitably leads to a marked temperature rise of $\mathrm{Si}$ nanostructures, which are required to withstand large thermal stresses for maintaining their proper functions. Si nanostructures are also the building blocks for many novel nanotechnology applications, including energy harvesting and storage, flexible and stretchable electronics, sensors and nanoelectromechanical systems.[1] The reliability concerns of these applications call for a fundamental understanding of the mechanical behavior of Si nanostructures at elevated temperatures. Here we report the in situ tensile testing of single crystal Si NWs in the temperature range of RT to $600 \mathrm{~K}$.[2] We employ a newly developed microelectromechanical system (MEMS)[3-6] for conducting the nano-thermomechanical testing inside a transmission electron microscope (TEM). This platform allows stress-strain measurement with simultaneous TEM imaging of atomic-scale deformation at different temperatures.[2,7] An on-chip heater is built into the MEMS-based platform, allowing the controlled heating of the specimen.

Single crystal Si NWs were synthesized by chemical evaporation deposition through the vapor-liquidsolid mechanism. The Si NWs used for mechanical testing are $\langle 112\rangle$-oriented and have a central $\{111\}$ twin boundary running parallel to the axial direction of the NW. In situ TEM tensile testing of individual Si NWs was performed at temperatures ranging from 295 to $600 \mathrm{~K}$. Fig. 1 reveals that the Si NWs under tension are brittle at RT, but exhibit pronounced plastic deformation followed by fracture at elevated temperatures (e.g., $600 \mathrm{~K}$ ). Fig.1a shows the stress-strain curve of a Si NW with a diameter of $61 \mathrm{~nm}$ at $295 \mathrm{~K}$. This NW initially exhibited a linear elastic response. As the tensile strain increased to $7.4 \%$ and the corresponding tensile stress to $11.5 \mathrm{GPa}$, brittle fracture occurred. As shown in Fig. 1b, the tensile stress-strain curve was initially linear and became slightly nonlinear with increasing stress, until the maximum stress of $5.8 \mathrm{GPa}$ was reached at the critical strain of $4.0 \%$. Subsequently, the stress decreased with increasing strain, and this softening response continued until fracture occurred at the strain of $4.7 \%$. The inset in Fig. 1b shows the TEM image of fracture surfaces, which exhibit the features of ductile failure such as localized necking, deformation bands and rough fracture planes. Figure 1c shows the TEM snapshots during in situ tensile testing at $600 \mathrm{~K}$, and the corresponding stress/strain for each snapshot is marked in Fig. 1b. It is seen that the maximum stress and ensuing softening response can be associated with surface nucleation and migration of dislocations as well as dislocation interactions with the twin boundary, as marked by blue arrows in Fig. 1c ii-iv. These continued dislocation processes resulted in large plastic strains in Si NWs at elevated temperatures. We also found that a decrease in the NW diameter can lead to enhanced plastic deformation. Fig. 1b includes the tensile stress-strain curve of a thinner Si NW with a diameter of $24 \mathrm{~nm}$ at $600 \mathrm{~K}$, which is qualitatively similar to the Si NW with a diameter of 66 $\mathrm{nm}$ at $600 \mathrm{~K}$. However, in this thinner $\mathrm{NW}$, a lower maximum stress of $5.1 \mathrm{GPa}$ was attained at a smaller critical strain of $4.3 \%$, signifying a stronger tendency to plastic deformation. Moreover, the subsequent softening response was sustained over a strain range of $1.6 \%$ until the fracture strain $\varepsilon$ of $5.9 \%$; this strain range is defined as plastic strain $\varepsilon_{\mathrm{p}}$. Hence, both $\varepsilon_{\mathrm{p}}$ and $\varepsilon_{\mathrm{f}}$ measured for the thinner Si NW are larger than the corresponding $\varepsilon_{\mathrm{p}}$ of $0.7 \%$ and $\varepsilon$ f of $4.7 \%$ for the thicker Si NW (Fig. 1b). The more extensive plasticity 
in the thinner Si NW was manifested by the formation of a number of deformation bands that are uniformly distributed throughout the entire NW, which contrasts with fewer deformation bands in the thicker NW (Fig. 1c).

To further understand the size and temperature dependence of dislocation-mediated plasticity and fracture, we tested $78 \mathrm{Si}$ NWs with the diameter range of 24-160 nm and in the temperature range of 295$600 \mathrm{~K}$. As shown in Fig. 2a, the plastic strain $\varepsilon_{\mathrm{p}}$ increases with decreasing NW diameter. For example, at $600 \mathrm{~K}, \varepsilon_{\mathrm{p}}$ increases from 0.27 to $1.6 \%$, as the NW diameter decreases from 160 to $24 \mathrm{~nm}$. For the same diameter, $\varepsilon_{\mathrm{p}}$ increases with temperature. The results of the $78 \mathrm{Si}$ NWs tested are summarized in a deformation mechanism map of Fig. 2b. In this map, green circles represent Si NWs with dislocationmediated failure, while black squares Si NWs with brittle cleavage fracture. A transition line, as defined by a critical $\varepsilon_{p}$ of $0.1 \%$, is drawn to separate the regimes of brittle and dislocation-mediated responses. The map also reveals that as the NW diameter decreases, the critical temperature associated with dislocation-mediated plastic deformation decreases. For example, the Si NW with diameter of $110 \mathrm{~nm}$ showed brittle fracture at $398 \mathrm{~K}$, while the NW with diameter of $30 \mathrm{~nm}$ exhibited plastic deformation with dislocation activities even at $362 \mathrm{~K}$. Hence, Fig. $2 \mathrm{~b}$ indicates that both the increase of temperature and the decrease of NW diameter can promote plastic deformation in Si NWs, leading to dislocation-mediated failure.[8]

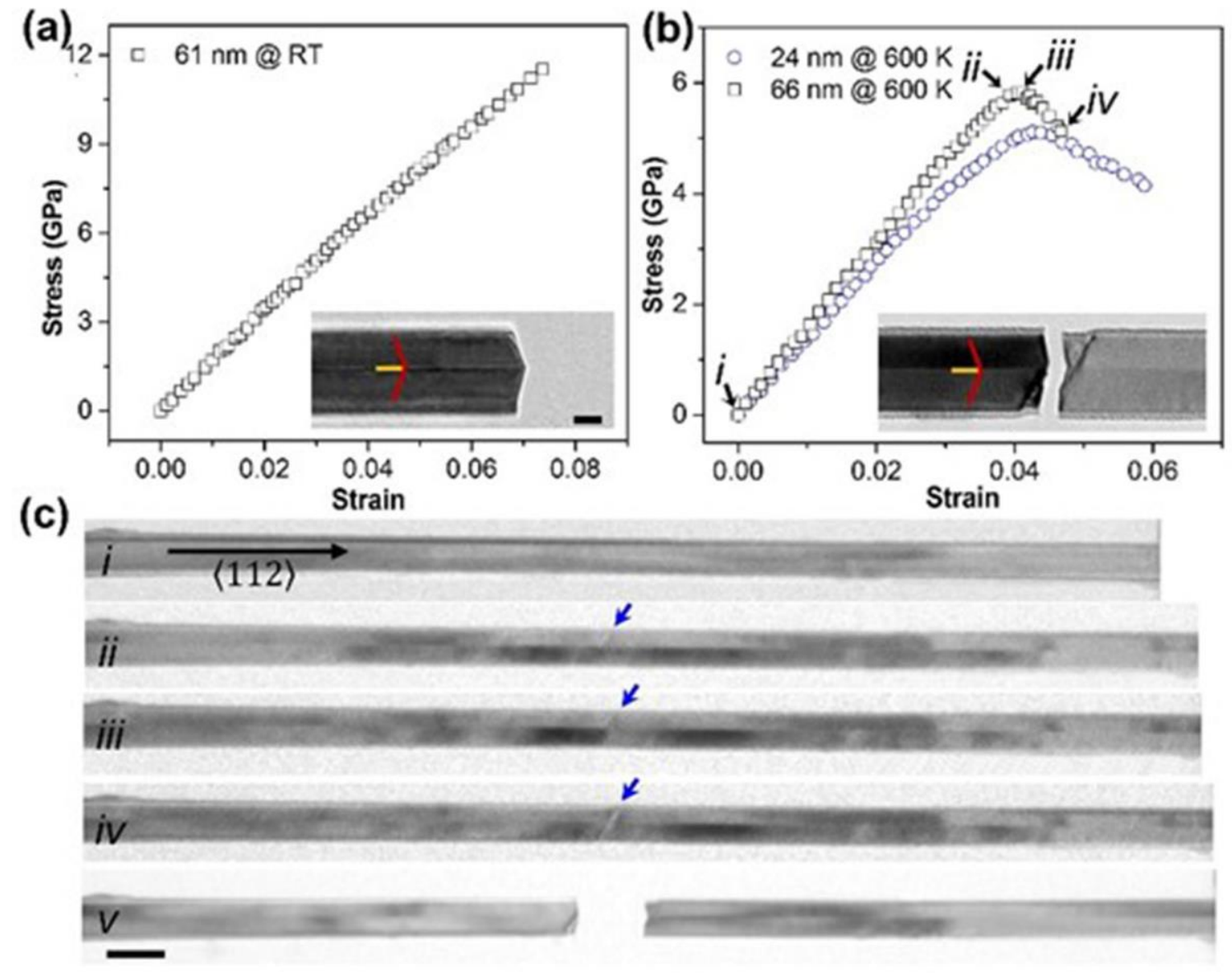

Figure 1. In situ MEMS-based measurement and TEM observation of mechanical behavior of individual Si NWs under uniaxial tension at room and elevated temperatures. 

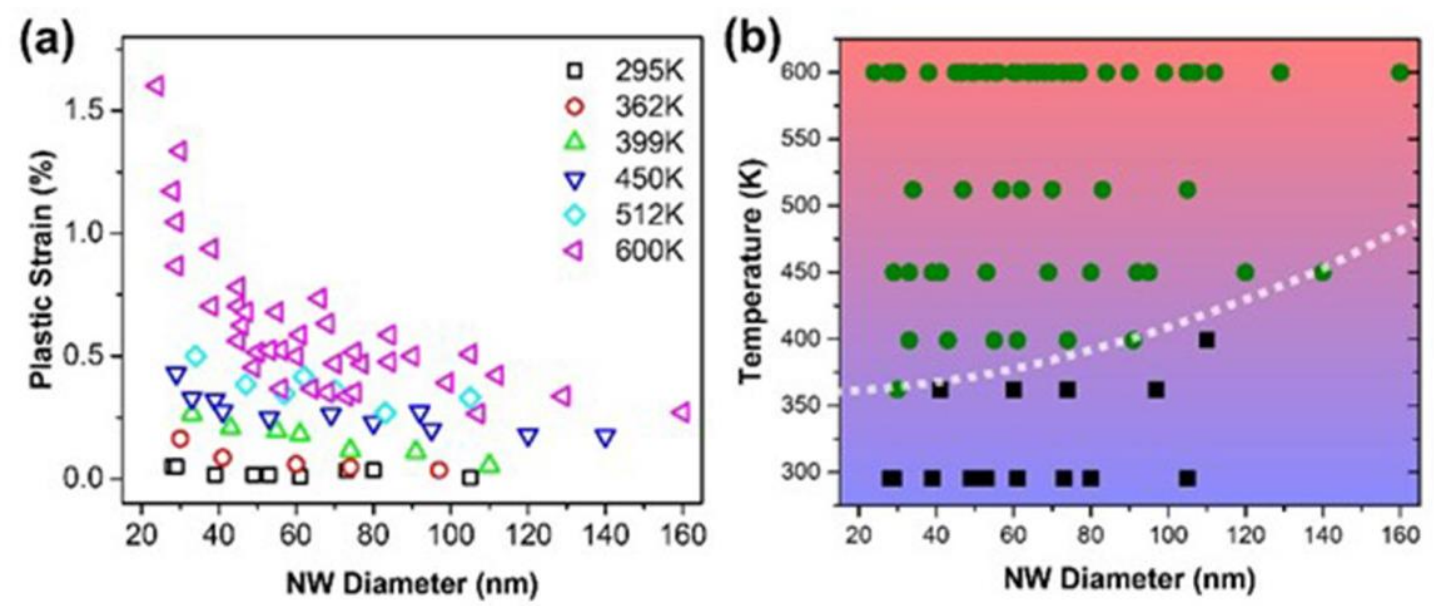

Figure 2. Size and temperature dependence of brittle versus ductile behaviors of $78 \mathrm{Si}$ NWs tested in the temperature range of 295 to $600 \mathrm{~K}$.

References

[1] F. Xu, W. Lu, and Y. Zhu, Acs Nano 5, 672 (2011).

[2] G. Cheng, Y. Zhang, T.-H. Chang, Q. Liu, L. Chen, W. D. Lu, T. Zhu, and Y. Zhu, Nano Letters 19, 5327 (2019).

[3] G. M. Cheng, S. Yin, T. H. Chang, G. Richter, H. J. Gao, and Y. Zhu, Physical Review Letters 19, 256101, 256101 (2017).

[4] S. Yin, G. Cheng, G. Richter, H. Gao, and Y. Zhu, ACS nano 13, 9082 (2019).

[5] T. H. Chang, G. M. Cheng, C. J. Li, and Y. Zhu, Extreme Mechanics Letters 8, 177 (2016).

[6] S. Yin, G. Cheng, T.-H. Chang, G. Richter, Y. Zhu, and H. Gao, Nature communications 10, 2004 (2019).

[7] T.-H. Chang and Y. Zhu, Applied Physics Letters 103, 263114 (2013).

[8] The authors acknowledge funding from the National Science Foundation (NSF) under Award No. CMMI-1762511, and the use of the Analytical Instrumentation Facility (AIF) at North Carolina State University (NSF Award No. ECCS-1542015). 